\title{
Chiral Perturbation Theory Analysis of the Baryon Magnetic Moments
}

\author{
Elizabeth Jenkins, ${ }^{a *}$ Michael Luke, ${ }^{b}$ \\ Aneesh V. Manohar, ${ }^{a *}$ and Martin J. Savage ${ }^{b \dagger}$ \\ a) CERN TH Division, CH-1211 Geneva 23, Switzerland \\ b) Department of Physics, University of California at San Diego, \\ 9500 Gilman Drive, La Jolla, CA 92093
}

\begin{abstract}
Nonanalytic $m_{q}^{1 / 2}$ and $m_{q} \ln m_{q}$ chiral corrections to the baryon magnetic moments are computed. The calculation includes contributions from both intermediate octet and decuplet baryon states. Unlike the one-loop contributions to the baryon axial currents and masses, the contribution from decuplet intermediate states does not partially cancel that from octet intermediate states. The fit to the observed magnetic moments including $m_{q}^{1 / 2}$ corrections is found to be much worse than the tree level $S U(3)$ fit if values for the baryon-pion axial coupling constants obtained from a tree level extraction are used. Using the axial coupling constant values extracted at one loop results in a better fit to the magnetic moments than the tree level $S U(3)$ fit. There are three linear relations amongst the magnetic moments when $m_{q}^{1 / 2}$ corrections are included, and one relation including $m_{q}^{1 / 2}, m_{q} \ln m_{q}$ and $m_{q}$ corrections. These relations are independent of the axial coupling constants of the baryons and agree well with experiment.
\end{abstract}

CERN-TH.6735/92

UCSD/PTH 92-34

hep-ph/9212226

December 1992

\footnotetext{
* On leave from the University of California at San Diego.

$\dagger$ SSC Fellow
} 
The baryon magnetic moments were first predicted theoretically on the basis of flavor $S U(3)$ symmetry [1]. The Coleman-Glashow $S U(3)$ relations yield the octet baryon magnetic moments in terms of two parameters. These relations are easily derived from the Lagrangian

$$
\mathcal{L}=\frac{e}{4 m_{N}}\left(\mu_{D} \operatorname{Tr} \bar{B}_{v} \sigma_{\mu \nu} F^{\mu \nu}\left\{Q, B_{v}\right\}+\mu_{F} \operatorname{Tr} \bar{B}_{v} \sigma_{\mu \nu} F^{\mu \nu}\left[Q, B_{v}\right]\right)
$$

where the parameters $\mu_{D, F}$ multiply the two independent $S U(3)$ invariants, $m_{N}$ is the nucleon mass, and

$$
Q=\left(\begin{array}{ccc}
\frac{2}{3} & 0 & 0 \\
0 & -\frac{1}{3} & 0 \\
0 & 0 & -\frac{1}{3}
\end{array}\right)
$$

is the charge matrix for the three light quarks $u, d$ and $s$. Calculation of the magnetic moments beyond tree approximation is possible in chiral perturbation theory [2]. The leading corrections which occur at one loop have a non-analytic dependence on the light quark masses of the form $m_{q}^{1 / 2}$ and $m_{q} \ln m_{q}$. The $m_{q}^{1 / 2}$ and $m_{q} \ln m_{q}$ terms for the baryon magnetic moments are calculable since they are non-analytic in the quark masses [3] [4] and therefore can not arise from terms in the chiral Lagrangian with additional insertions of the quark mass matrix. The $m_{q}^{1 / 2}$ contribution [2] [5] and $m_{q} \ln m_{q}$ contribution [6] from intermediate octet states have been computed previously. Recent work on baryon chiral perturbation theory [7]-[1] indicates that the spin-3/2 decuplet of baryons contributes significantly as an intermediate state in one-loop diagrams with octet baryon initial and final states. In this letter, we calculate the $m_{q}^{1 / 2}$ and $m_{q} \ln m_{q}$ contributions to the baryon magnetic moments including both intermediate octet and intermediate decuplet states.

The chiral Lagrangian for baryon fields depends on the pseudoscalar pion octet

$$
\pi=\frac{1}{\sqrt{2}}\left(\begin{array}{ccc}
\frac{1}{\sqrt{2}} \pi^{0}+\frac{1}{\sqrt{6}} \eta & \pi^{+} & K^{+} \\
\pi^{-} & -\frac{1}{\sqrt{2}} \pi^{0}+\frac{1}{\sqrt{6}} \eta & K^{0} \\
K^{-} & \bar{K}^{0} & -\frac{2}{\sqrt{6}} \eta
\end{array}\right)
$$

which couples to the baryon matter fields through the vector and axial vector combinations

$$
V^{\mu}=\frac{1}{2}\left(\xi \partial^{\mu} \xi^{\dagger}+\xi^{\dagger} \partial^{\mu} \xi\right), \quad A^{\mu}=\frac{i}{2}\left(\xi \partial^{\mu} \xi^{\dagger}-\xi^{\dagger} \partial^{\mu} \xi\right)
$$

where

$$
\xi=e^{i \pi / f}, \quad \Sigma=\xi^{2}=e^{2 i \pi / f}
$$


and $f \approx 93 \mathrm{MeV}$ is the pion decay constant. Under $S U(3)_{L} \times S U(3)_{R}$ chiral symmetry,

$$
\begin{aligned}
& \Sigma \rightarrow L \Sigma R^{\dagger}, \quad \xi \rightarrow L \xi U^{\dagger}=U \xi R^{\dagger}, \\
& B \rightarrow U B U^{\dagger}, \quad T_{a b c}^{\mu} \rightarrow U_{a}^{d} U_{b}^{e} U_{c}^{f} T_{d e f}^{\mu},
\end{aligned}
$$

where $U$ is defined by the transformation of $\xi$, and $B$ and $T^{\mu}$ denote the baryon octet and decuplet fields, respectively. A consistent chiral derivative expansion for baryon fields [7] can be written in terms of velocity-dependent baryon fields,

$$
\begin{aligned}
& T_{v}^{\mu}(x)=e^{i m_{B} \psi v_{\mu} x^{\mu}} T^{\mu}(x), \\
& B_{v}(x)=e^{i m_{B} \psi v_{\mu} x^{\mu}} B(x),
\end{aligned}
$$

where $m_{B}$ is the $S U(3)$ invariant mass of the octet baryon multiplet. The lowest order chiral Lagrangian for octet and decuplet baryons is

$$
\begin{aligned}
L_{v}^{0} & =i \operatorname{Tr} \bar{B}_{v}(v \cdot \mathcal{D}) B_{v}+2 D \operatorname{Tr} \bar{B}_{v} S_{v}^{\mu}\left\{A_{\mu}, B_{v}\right\}+2 F \operatorname{Tr} \bar{B}_{v} S_{v}^{\mu}\left[A_{\mu}, B_{v}\right] \\
& -i \bar{T}_{v}^{\mu}(v \cdot \mathcal{D}) T_{v \mu}+\mathcal{C}\left(\bar{T}_{v}^{\mu} A_{\mu} B_{v}+\bar{B}_{v} A_{\mu} T_{v}^{\mu}\right)+2 \mathcal{H} \bar{T}_{v}^{\mu} S_{v \nu} A^{\nu} T_{v \mu} \\
& +\delta \bar{T}_{v}^{\mu} T_{v \mu}+\frac{f^{2}}{4} \operatorname{Tr} \partial_{\mu} \Sigma \partial^{\mu} \Sigma^{\dagger},
\end{aligned}
$$

where the decuplet-octet mass difference $\delta=m_{T}-m_{B}$, and $m_{T}$ is the $S U(3)$ invariant mass of the baryon decuplet. The vector combination of pion fields appears in Eq. (8) through the covariant derivatives

$$
\begin{aligned}
& \mathcal{D}^{\nu} B=\partial^{\nu} B+\left[V^{\nu}, B\right] \\
& \mathcal{D}^{\nu} T_{a b c}^{\mu}=\partial^{\nu} T_{a b c}^{\mu}+\left(V^{\nu}\right)_{a}^{d} T_{d b c}^{\mu}+\left(V^{\nu}\right)_{b}^{d} T_{a d c}^{\mu}+\left(V^{\nu}\right)_{c}^{d} T_{a b d}^{\mu} .
\end{aligned}
$$

The axial vector pion couplings are described by four coupling constants $D, F, \mathcal{C}$ and $\mathcal{H}$. The octet and decuplet baryon propagators obtained from this Lagrangian are $i /(v \cdot k)$ and $i P_{v}^{\mu \nu} /(v \cdot k-\delta)$, where

$$
P_{v}^{\mu \nu}=\sum_{i} \mathcal{U}_{i}^{\mu} \overline{\mathcal{U}}_{i}^{\nu}=\left(v^{\mu} v^{\nu}-g^{\mu \nu}\right)-\frac{4}{3} S_{v}^{\mu} S_{v}^{\nu}
$$

is a polarization projector for the spin-3/2 Rarita-Schwinger decuplet field. The decuplet field satisfies the constraint $v \cdot T=0$.

The calculation of the baryon magnetic moments involves electromagnetic couplings. Electromagnetism is incorporated into Lagrangian (8) by making the following substitutions,

$$
\begin{aligned}
& V^{\mu} \rightarrow V^{\mu}+\frac{1}{2} i e \mathcal{A}^{\mu}\left(\xi^{\dagger} Q \xi+\xi Q \xi^{\dagger}\right), \\
& A^{\mu} \rightarrow A^{\mu}-\frac{1}{2} e \mathcal{A}^{\mu}\left(\xi Q \xi^{\dagger}-\xi^{\dagger} Q \xi\right),
\end{aligned}
$$


and

$$
\partial_{\mu} \Sigma \rightarrow \mathcal{D}_{\mu} \Sigma=\partial_{\mu} \Sigma+i e \mathcal{A}_{\mu}[Q, \Sigma]
$$

where $\mathcal{A}_{\mu}$ is the photon field. The octet baryon magnetic moment Lagrangian is given in Eq. (1); the full chiral structure of the operator is given by the replacement

$$
Q \rightarrow \frac{1}{2}\left(\xi Q \xi^{\dagger}+\xi^{\dagger} Q \xi\right)
$$

The one loop corrections also involve the decuplet magnetic moment and the decupletoctet transition magnetic moment. There is only one $S U(3)$ invariant in the tensor product $\overline{\mathbf{1 0}} \otimes \mathbf{1 0} \otimes \mathbf{8}$, which can be chosen to be proportional to the charge, so the decuplet magnetic moment operator can be written in the form

$$
\mathcal{L}=-i \frac{e}{m_{N}} \mu_{C} q_{i} \bar{T}_{v i}^{\mu} T_{v i}^{\nu} F_{\mu \nu}
$$

where $q_{i}$ is the charge of the $\imath^{\text {th }}$ element of the decuplet, and the operator is normalized so that the magnetic moment of the $\imath^{\text {th }}$ state is $q_{i} \mu_{C}$ nuclear magnetons. The measured value of the $\Omega^{-}$magnetic moment [12] determines $\mu_{C}=1.94 \pm 0.22$. The octet-decuplet transition magnetic moment operator has the form [13]

$$
\mathcal{L}=i \frac{e}{2 m_{N}} \mu_{T} F_{\mu \nu}\left(\epsilon_{i j k} Q_{l}^{i} \bar{B}_{v m}^{j} S_{v}^{\mu} T_{v}^{\nu k l m}+\epsilon^{i j k} Q_{i}^{l} \bar{T}_{v k l m}^{\mu} S_{v}^{\nu} B_{v j}^{m}\right)
$$

where $i, j, k, l, m$ are $S U(3)$ flavor indices. The octet-decuplet transition electric quadrupole moment operator has an additional derivative, and therefore is higher order in the chiral expansion. The Lagrangian Eq. (15) implies that the $\Delta \rightarrow N \gamma$ helicity amplitudes $A_{3 / 2}$ and $A_{1 / 2}$ are in the ratio $\sqrt{3}: 1$, in good agreement with experiment 14$]$ [15. The measured values of the helicity amplitudes determine $\mu_{T}=-7.7 \pm 0.5$.

In addition to the electromagnetic couplings, the calculation of the magnetic moments to nonleading order requires the introduction of $S U(3)$ breaking through the quark mass matrix $\mathcal{M}=\operatorname{diag}\left(m_{u}, m_{d}, m_{s}\right)$. At leading order in the quark mass expansion, the pseudoGoldstone bosons acquire non-vanishing masses and the $S U(3)$ baryon multiplets are no longer degenerate.

The calculation of the baryon magnetic moments presented here includes the treelevel Coleman-Glashow formulæ and the leading non-analytic correction arising from the 
diagrams displayed in fig. 1 and fig. 2.1. The magnetic moments (in units of nuclear magnetons) can be written in the form

$$
\begin{array}{r}
\mu_{i}=\alpha_{i}+\sum_{X=\pi, K} \beta_{i}^{(X)} \frac{M_{X} m_{N}}{8 \pi f^{2}}+\sum_{X=\pi, K} F\left(M_{X}, \delta, \mu\right) \beta_{i}^{\prime(X)} \frac{m_{N}}{8 \pi f^{2}} \\
+\sum_{X=\pi, K, \eta} \frac{1}{32 \pi^{2} f^{2}}\left(\bar{\gamma}_{i}^{(X)}-2 \bar{\lambda}_{i}^{(X)} \alpha_{i}\right) M_{X}^{2} \ln M_{X}^{2} / \mu^{2}
\end{array}
$$

where $\alpha_{i}$ are the tree-level predictions derived from Lagrangian (1),$\beta_{i}^{(\pi, K)}$ and $\beta_{i}^{\prime(\pi, K)}$ are the contributions from pion and kaon loops from fig. 1 with intermediate octet and decuplet states respectively, $\bar{\gamma}_{i}^{(\pi, K, \eta)}$ are the contributions from pion, kaon and $\eta$ loops from fig. 2, and $\bar{\lambda}_{i}$ is the wavefunction renormalization contribution. $\eta$ loops do not contribute to the diagrams of fig. 1 since the $\eta$ is neutral. The scale $\mu$ is an arbitrary renormalization scale, and is chosen to be $\mu \sim 1 \mathrm{GeV}$. The chiral coefficients $\alpha_{i}, \beta_{i}^{(X)}, \beta_{i}^{\prime(X)}, \bar{\gamma}_{i}^{(X)}$, and $\bar{\lambda}_{i}^{(X)}$ are listed in Appendix A. The pion contribution to the $m_{q}^{1 / 2}$ terms is comparable to the kaon contribution, and cannot be neglected. The pion mass is not large compared with the $\Delta-N$ mass difference $\delta$, so the full dependence of the Feynman graph on the ratio $\delta / M_{\pi}$ must be retained. This dependence is described by the function $F\left(M_{X}, \delta, \mu\right)$, which is given explicitly in the appendix. The function $F$ is normalized so that $F\left(M_{X}, 0, \mu\right)=M_{X}$. The dependence of $F$ on the renormalization scale $\mu$ is of the form $\delta \ln \mu^{2}$. The $\mu$ dependence of the $\beta$ terms in Eq. (16) is compensated by the $\mu$ dependence of the $m_{q}$ independent $\alpha$ terms.2 The $\mu$ dependence of the $m_{q} \ln m_{q}$ terms in Eq. (16) is canceled by the $\mu$ dependence of local counterterms. These counterterms are the most general invariants that can be constructed out of $B, \bar{B}, M$ and $Q F_{\mu \nu}$ which are linearly independent and preserve parity and time-reversal invariance,

$$
\begin{aligned}
\mathcal{L}=F_{\mu \nu}( & c_{1} \operatorname{Tr} \bar{B} M Q \sigma^{\mu \nu} B+c_{2} \operatorname{Tr} \bar{B} Q \sigma^{\mu \nu} B M+c_{3} \operatorname{Tr} \bar{B} \sigma^{\mu \nu} B M Q \\
& +c_{4} \operatorname{Tr} \bar{B} M \sigma^{\mu \nu} B Q+c_{5} \operatorname{Tr} \bar{B} \sigma^{\mu \nu} B \operatorname{Tr} M Q \\
& \left.+c_{6} \operatorname{Tr} M \operatorname{Tr} \bar{B} Q \sigma^{\mu \nu} B+c_{7} \operatorname{Tr} M \operatorname{Tr} B \sigma^{\mu \nu} B Q\right) .
\end{aligned}
$$

1 There are also graphs which involve the $\bar{B} B \pi \mathcal{A}_{\mu}$ vertex arising from the $Q$ terms in Eq. (11). These graphs do not contribute to the magnetic moments.

2 The $\mu$ dependence of the $\beta$ terms can only be absorbed into the $\alpha$ terms if the $\delta$ dependence of both the pion and kaon loops is retained, since $\delta$ is an $S U(3)$ singlet mass parameter. The mass difference $\delta$ also affects the $m_{q} \ln m_{q}$ terms in Eq. (16). However, for these terms, the pion loop contributions are negligible relative to the kaon and eta contributions, so we have chosen not to compute the analogous function, and have combined the octet and decuplet contributions into a single coefficient. 
The counterterm with flavor structure $\operatorname{Tr} \bar{B} Q \operatorname{Tr} B M+\operatorname{Tr} \bar{B} M \operatorname{Tr} B Q$ is a linear combination of the counterterms in Eq. (17), and the counterterm $i(\operatorname{Tr} \bar{B} Q \operatorname{Tr} B M-\operatorname{Tr} \bar{B} M \operatorname{Tr} B Q)$ violates time-reversal invariance.

The theoretical computation can now be compared with experiment. There are seven octet magnetic moments as well as the $\Sigma^{0} \rightarrow \Lambda \gamma$ transition magnetic moment which are measured experimentally. (The $\Sigma^{0}$ magnetic moment has not been measured.) The wellknown $S U(3)$ symmetric fit to the data is obtained by using only the tree level $\alpha_{i}$ terms in Eq. (16), and has an average deviation between the theoretical and experimental numbers of 0.25 nuclear magnetons. There are eight experimentally measured magnetic moments, and two parameters $\mu_{D}$ and $\mu_{F}$, which leads to the six linear relations found by Coleman and Glashow [1]

$$
\begin{array}{ll}
\mu_{\Sigma^{+}}=\mu_{p}(2.42 \pm 0.05=2.79), & \mu_{\Sigma^{-}}+\mu_{n}=-\mu_{p}(-3.07 \pm 0.03=-2.79) \\
2 \mu_{\Lambda}=\mu_{n}(-1.23 \pm 0.01=-1.91), & \mu_{\Xi^{-}}=\mu_{\Sigma^{-}}(-0.65=-1.16 \pm 0.03) \\
\mu_{\Xi^{0}}=\mu_{n}(-1.25 \pm 0.01=-1.91), & 2 \mu_{\Lambda \Sigma^{0}}=-\sqrt{3} \mu_{n}(3.22 \pm 0.16=3.31)
\end{array}
$$

where the relations are written so that all terms in a given relation have the same sign. The experimental numbers for each relation are given in parentheses, and have been rounded off to two decimal digits.

The $m_{q}^{1 / 2}$ correction is more important than the $m_{q} \ln m_{q}$ contribution and the seven $\mathcal{O}\left(m_{q}\right)$ counterterms. We therefore first discuss the theoretical predictions including only the $m_{q}^{1 / 2}$ corrections, which have the same two unknown parameters $\mu_{D}$ and $\mu_{F}$ as the tree level predictions. There are three relations between the magnetic moments which are valid to $\mathcal{O}\left(m_{q}^{1 / 2}\right)$, irrespective of the baryon-pion axial coupling constants. These relations, linear combinations of the six relations of Eq. (18) valid at tree level, were noted by Caldi and Pagels [2] and are also valid when decuplet graphs are included:

$$
\begin{aligned}
& \mu_{\Sigma^{+}}=-2 \mu_{\Lambda}-\mu_{\Sigma^{-}}(2.42 \pm 0.05=2.39 \pm 0.03) \\
& \mu_{\Xi^{0}}+\mu_{\Xi^{-}}+\mu_{n}=2 \mu_{\Lambda}-\mu_{p}(-3.81 \pm 0.0=-4.02 \pm 0.01) \\
& \mu_{\Lambda}-\sqrt{3} \mu_{\Lambda \Sigma^{0}}=\mu_{\Xi^{0}}+\mu_{n}(-3.40 \pm 0.14=-3.16 \pm 0.01) .
\end{aligned}
$$

The experimental values are shown in parentheses. These relations are in good agreement with experiment, and work much better than the tree level relations Eq. (18). The remaining three relations are predictions for the deviation from any three of the Coleman-Glashow 
relations, e.g.

$$
\begin{aligned}
& \mu_{p}-\mu_{\Sigma^{+}}=0.37 \pm 0.05= \\
& \quad \frac{m_{N}}{8 \pi f^{2}}\left[\left(\frac{1}{3} D^{2}+2 D F-F^{2}\right)\left(M_{K}-M_{\pi}\right)+\frac{5}{18} \mathcal{C}^{2}\left(F\left(M_{K}, \delta, \mu\right)-F\left(M_{\pi}, \delta, \mu\right)\right)\right], \\
& \mu_{\Xi^{-}-}-\mu_{\Sigma^{-}}=0.51 \pm 0.03= \\
& \quad \frac{m_{N}}{8 \pi f^{2}}\left[\left(-\frac{1}{3} D^{2}+2 D F+F^{2}\right)\left(M_{K}-M_{\pi}\right)+\frac{1}{18} \mathcal{C}^{2}\left(F\left(M_{K}, \delta, \mu\right)-F\left(M_{\pi}, \delta, \mu\right)\right)\right], \\
& \mu_{\Xi^{0}-\mu_{n}}=0.66 \pm 0.01= \\
& \quad \frac{m_{N}}{8 \pi f^{2}}\left[\left(2 D^{2}+2 F^{2}\right)\left(M_{K}-M_{\pi}\right)+\frac{1}{9} \mathcal{C}^{2}\left(F\left(M_{K}, \delta, \mu\right)-F\left(M_{\pi}, \delta, \mu\right)\right)\right],
\end{aligned}
$$

The numerical values in Eq. (20) are taken from experiment. The relations Eq. (20) are independent of the renormalization scale $\mu$, which cancels in the difference $F\left(M_{K}, \delta, \mu\right)-$ $F\left(M_{\pi}, \delta, \mu\right)$. The theoretical predictions using the tree level values for the axial couplings do not agree at all with the experimental data. The theoretical values for the relations in Eq. (20) are 1.79, 1.30, and 2.94 nuclear magnetons respectively, which are much larger than the experimental numbers. A least squares fit to the eight experimentally measured moments including $m_{q}^{1 / 2}$ corrections has an average deviation of 0.8 nuclear magnetons, which is more than three times larger than the tree level fit.

Naively, one might conclude that the reason for this failure is that the $K$ mass is too large for chiral perturbation theory to be valid and $K$ meson loops should be omitted, or that intermediate decuplet states should not be included in chiral perturbation theory. Neither of these two conclusions is substantiated by the data. One can repeat the fit using Eq. (16) without including the decuplet contributions and the fit to experiment is still much worse than the tree level fit, the average deviation from experiment being 0.7 nuclear magnetons. One can also repeat the fits dropping the $K$ loops completely and retaining only the pion loops; the average deviation from experiment is 0.7 nuclear magnetons if the decuplet is included, and 0.6 nuclear magnetons if it is omitted. All of these fits are much worse than the tree level fit, which has neither pion nor kaon loops. The pion mass is small enough that chiral perturbation theory in the pion mass is valid, so there is no theoretical reason why pion loops should be dropped from the calculation. Pion loop contributions alone are in serious disagreement with experiment.

We believe that the reason for the disagreement is that the formula Eq. (16) overestimates the size of kaon loops. The suppression of kaon loops in chiral perturbation

3 We have used the best fit values $F=0.47$ and $D=0.81$ from [16] and $\mathcal{C}=-1.53$ from [9]. 
theory has been discussed before by Gasser and Leutwyler [4]. They have suggested a method for evaluating loop graphs that suppresses kaon loops, which they call improved chiral perturbation theory (ICPT). There is some empirical evidence that kaon loops are suppressed. Loop corrections to the baryon masses and hyperon non-leptonic decays [8] work well if the one-loop corrected values for the axial vector coupling constants [7] (which are smaller than the tree level values) are used. A similar result is found for the nucleon polarizability [10]. The one-loop couplings $D, F$, and $\mathcal{C}$ are proportional to, but smaller than, their tree level values. Another well known effect which also suppresses kaon loops is that $f_{K}=1.2 f_{\pi}$. We have therefore computed the results using the central values for the axial couplings extracted at one loop $D=0.61, F=0.4$ and $\mathcal{C}=-1.2$ given in [9] and [13], and $f_{K}=1.2 f_{\pi}$. The deviations from the Coleman-Glashow relations are now 0.59, 0.50 and 1.0 nuclear magnetons, and are much closer to the experimental values. One can also do a least squares fit to the data, which has the same two unknown parameters as the tree level fit. The average deviation is 0.11 nuclear magnetons, about half that of the tree level fit.

Finally, one can include the $m_{q} \ln m_{q}$ contributions to the baryon magnetic moments. Since $\ln M_{K}^{2} / \mu^{2}$ is not very big, the $m_{q} \ln m_{q}$ terms are not expected to be significantly enhanced relative to the counterterms in Eq. (17), so we will include the counterterms in the theoretical predictions. There are seven unknown counterterm coefficients $c_{1}-c_{7}$, as well as the two tree level parameters $\mu_{D}$ and $\mu_{F}$. The $c_{6}$ and $c_{7}$ counterterms have the same vector $S U(3)$ structure as the lowest order terms, so there are effectively seven independent operators, and there exists one linear relation amongst the baryon magnetic moments which is valid including all terms of order $m_{q}^{1 / 2}, m_{q} \ln m_{q}$ and $m_{q}$,

$$
6 \mu_{\Lambda}+\mu_{\Sigma^{-}}-4 \sqrt{3} \mu_{\Lambda \Sigma^{0}}=4 \mu_{n}-\mu_{\Sigma^{+}}+4 \mu_{\Xi^{0}}(-15.99 \pm 0.56=-15.07 \pm 0.08)
$$

which agrees well with experiment. In addition to the relations of Eqs. (18)-(21), there is also the $S U(2)$ relation

$$
\mu_{\Sigma^{+}}+\mu_{\Sigma^{-}}=2 \mu_{\Sigma^{0}}
$$

which cannot be tested because the $\Sigma^{0}$ magnetic moment has not been measured.

In conclusion, we have calculated the nonanalytic contributions proportional to $m_{q}^{1 / 2}$ and $m_{q} \ln m_{q}$ to the baryon magnetic moments in chiral perturbation theory. We find that the one-loop fit to the data with the $m_{q}^{1 / 2}$ corrections is much worse than the tree-level 
result if the tree level axial couplings are used, but is better than the tree level result if the one loop axial couplings are used. This suggests that kaon loop graphs are overestimated in chiral perturbation theory when the tree-level couplings are used at the vertices. Unlike the cases of the baryon axial couplings [7] and masses [8], including virtual baryon decuplet states does not give appreciably better agreement with the data. Relations amongst the magnetic moments which are independent of the axial couplings are found to be in good agreement with experiment.

\section{Acknowledgements}

This work was supported in part by the Department of Energy under grant number DOE-FG03-90ER40546. AVM was also supported by a National Science Foundation Presidential Young Investigator award PHY-8958081. MJS acknowledges support of a Superconducting Supercollider National Fellowship from the Texas National Research Laboratory Commission under grant FCFY9219. 


\section{Appendix A. Chiral Coefficients}

The tree level coefficients are:

$$
\begin{array}{lll}
\alpha_{p}=\frac{1}{3} \mu_{D}+\mu_{F}, & \alpha_{\Sigma^{+}}=\frac{1}{3} \mu_{D}+\mu_{F}, & \alpha_{\Xi^{0}}=-\frac{2}{3} \mu_{D}, \\
\alpha_{n}=-\frac{2}{3} \mu_{D}, & \alpha_{\Sigma^{0}}=\frac{1}{3} \mu_{D}, & \alpha_{\Xi^{-}}=\frac{1}{3} \mu_{D}-\mu_{F}, \\
\alpha_{\Lambda}=-\frac{1}{3} \mu_{D}, & \alpha_{\Sigma^{-}}=\frac{1}{3} \mu_{D}-\mu_{F}, & \alpha_{\Lambda \Sigma^{0}}=\frac{1}{\sqrt{3}} \mu_{D} .
\end{array}
$$

The one-loop coefficients $\beta_{i}$ and $\beta_{i}^{\prime}$ from the graphs in fig. 1 $(a)$ and $(b)$ are:

$$
\begin{array}{lll}
\beta_{p}^{(\pi)}=-(D+F)^{2}, & \beta_{\Sigma^{+}}^{(\pi)}=-\frac{2}{3} D^{2}-2 F^{2}, & \beta_{\Xi^{0}}^{(\pi)}=-(D-F)^{2}, \\
\beta_{n}^{(\pi)}=(D+F)^{2}, & \beta_{\Sigma^{0}}^{(\pi)}=0, & \beta_{\Xi^{-}}^{(\pi)}=(D-F)^{2}, \\
\beta_{\Lambda}^{(\pi)}=0, & \beta_{\Sigma^{-}}^{(\pi)}=\frac{2}{3} D^{2}+2 F^{2}, & \beta_{\Lambda \Sigma^{0}}^{(\pi)}=-\frac{4}{\sqrt{3}} D F, \\
\beta_{p}^{\prime(\pi)}=-\frac{2}{9} \mathcal{C}^{2}, & \beta_{\Sigma^{+}}^{(\pi)}=\frac{1}{18} \mathcal{C}^{2}, & \beta_{\Xi^{0}}^{(\pi)}=\frac{1}{9} \mathcal{C}^{2}, \\
\beta_{n}^{\prime(\pi)}=\frac{2}{9} \mathcal{C}^{2}, & \beta_{\Sigma^{0}}^{\prime(\pi)}=0, & \beta_{\Xi^{-}}^{\prime(\pi)}=-\frac{1}{9} \mathcal{C}^{2}, \\
\beta_{\Lambda}^{\prime(\pi)}=0, & \beta_{\Sigma^{-}}^{(\pi)}=-\frac{1}{18} \mathcal{C}^{2}, & \beta_{\Lambda \Sigma^{0}}^{\prime(\pi)}=-\frac{1}{3 \sqrt{3}} \mathcal{C}^{2},
\end{array}
$$

for the pion loops, and

$$
\begin{array}{lll}
\beta_{p}^{(K)}=-\frac{2}{3} D^{2}-2 F^{2}, & \beta_{\Sigma^{+}}^{(K)}=-(D+F)^{2}, & \beta_{\Xi^{0}}^{(K)}=(D+F)^{2}, \\
\beta_{n}^{(K)}=-(D-F)^{2}, & \beta_{\Sigma^{0}}^{(K)}=-2 D F, & \beta_{\Xi^{-}}^{(K)}=\frac{2}{3} D^{2}+2 F^{2}, \\
\beta_{\Lambda}^{(K)}=2 D F, & \beta_{\Sigma^{-}}^{(K)}=(D-F)^{2}, & \beta_{\Lambda \Sigma^{0}}^{(K)}=-\frac{2}{\sqrt{3}} D F, \\
\beta_{p}^{\prime(K)}=\frac{1}{18} \mathcal{C}^{2}, & \beta_{\Sigma^{+}}^{(K)}=-\frac{2}{9} \mathcal{C}^{2}, & \beta_{\Xi^{0}}^{(K)}=\frac{2}{9} \mathcal{C}^{2}, \\
\beta_{n}^{\prime(K)}=\frac{1}{9} \mathcal{C}^{2}, & \beta_{\Sigma^{0}}^{(K)}=-\frac{1}{6} \mathcal{C}^{2}, & \beta_{\Xi^{-}}^{(K)}=-\frac{1}{18} \mathcal{C}^{2}, \\
\beta_{\Lambda}^{\prime(K)}=\frac{1}{6} \mathcal{C}^{2}, & \beta_{\Sigma^{-}}^{\prime(K)}=-\frac{1}{9} \mathcal{C}^{2}, & \beta_{\Lambda \Sigma^{0}}^{(K)}=-\frac{1}{6 \sqrt{3}} \mathcal{C}^{2},
\end{array}
$$

for the graphs with kaon loops. The function $F(M, \delta, \mu)$ is

$$
\pi F(M, \delta, \mu)= \begin{cases}-\delta \ln M^{2} / \mu^{2}+2 \sqrt{M^{2}-\delta^{2}}\left[\frac{\pi}{2}-\tan ^{-1} \frac{\delta}{\sqrt{M^{2}-\delta^{2}}}\right] & \delta \leq M, \\ -\delta \ln M^{2} / \mu^{2}+\sqrt{\delta^{2}-M^{2}} \ln \frac{\delta-\sqrt{\delta^{2}-M^{2}}}{\delta+\sqrt{\delta^{2}-M^{2}}} & \delta>M .\end{cases}
$$

The wavefunction renormalization coefficients are $S U(2)$ invariant, and the values for the 
different $S U(2)$ multiplets are:

$$
\begin{array}{ll}
\bar{\lambda}_{N}^{(\pi)}=\frac{9}{4}(D+F)^{2}+2 \mathcal{C}^{2} & \bar{\lambda}_{\Sigma}^{(\pi)}=D^{2}+6 F^{2}+\frac{1}{3} \mathcal{C}^{2} \\
\bar{\lambda}_{\Lambda}(\pi)=3 D^{2}+\frac{3}{2} \mathcal{C}^{2} & \bar{\lambda}_{\Xi}^{(\pi)}=\frac{9}{4}(D-F)^{2}+\frac{1}{2} \mathcal{C}^{2} \\
\bar{\lambda}_{N}^{(K)}=\frac{5}{2} D^{2}-3 D F+\frac{9}{2} F^{2}+\frac{1}{2} \mathcal{C}^{2} & \bar{\lambda}_{\Sigma}^{(K)}=3 D^{2}+3 F^{2}+\frac{5}{3} \mathcal{C}^{2} \\
\bar{\lambda}_{\Lambda}^{(K)}=D^{2}+9 F^{2}+\mathcal{C}^{2} & \bar{\lambda}_{\Xi}^{(K)}=\frac{5}{2} D^{2}+3 D F+\frac{9}{2} F^{2}+\frac{3}{2} \mathcal{C}^{2} \\
\bar{\lambda}_{N}^{(\eta)}=\frac{1}{4}(D-3 F)^{2} & \bar{\lambda}_{\Sigma}^{(\eta)}=D^{2}+\frac{1}{2} \mathcal{C}^{2} \\
\bar{\lambda}_{\Lambda}^{(\eta)}=D^{2} & \bar{\lambda}_{\Xi}^{(\eta)}=\frac{1}{4}(D+3 F)^{2}+\frac{1}{2} \mathcal{C}^{2} \\
&
\end{array}
$$

The wavefunction renormalization coefficients for the transition magnetic moment are $\bar{\lambda}_{\Lambda \Sigma^{0}}^{(X)}=\frac{1}{2}\left(\bar{\lambda}_{\Lambda}^{(X)}+\bar{\lambda}_{\Sigma}^{(X)}\right)$, for $X=\pi, K, \eta$. The coefficients $\bar{\gamma}_{i}$ evaluated from the graphs in fig. 2 are:

$$
\begin{aligned}
& \bar{\gamma}_{p}^{(\pi)}=-\mu_{D}-\mu_{F}+\frac{1}{2}(D+F)^{2}\left(\mu_{D}-\mu_{F}\right)-\frac{32}{27} \mathcal{C}^{2} \mu_{C}+\frac{8}{9} \mathcal{C}(D+F) \mu_{T}, \\
& \bar{\gamma}_{n}^{(\pi)}=-(D+F)^{2} \mu_{F}+\frac{8}{27} \mathcal{C}^{2} \mu_{C}-\frac{8}{9} \mathcal{C}(D+F) \mu_{T}, \\
& \bar{\gamma}_{\Lambda}^{(\pi)}=-\frac{1}{3} \mu_{D}-\frac{2}{3} D^{2} \mu_{D}-\frac{2}{3} \mathcal{C} D \mu_{T} \\
& \bar{\gamma}_{\Sigma^{+}}^{(\pi)}=-\mu_{D}-\mu_{F}+\frac{2}{9}\left(D^{2}+6 D F-6 F^{2}\right) \mu_{D}-2 F^{2} \mu_{F}-\frac{2}{27} \mathcal{C}^{2} \mu_{C}+\frac{2}{9} \mathcal{C}(D+3 F) \mu_{T}, \\
& \bar{\gamma}_{\Sigma^{0}}^{(\pi)}=-\mu_{D}+\frac{2}{9}\left(D^{2}-6 F^{2}\right) \mu_{D}+\frac{4}{9} \mathcal{C} F \mu_{T}, \\
& \bar{\gamma}_{\Sigma^{-}}^{(\pi)}=-\mu_{D}+\mu_{F}+\frac{2}{9}\left(D^{2}-6 D F-6 F^{2}\right) \mu_{D}+2 F^{2} \mu_{F}+\frac{2}{27} \mathcal{C}^{2} \mu_{C}+\frac{2}{9} \mathcal{C}(F-D) \mu_{T}, \\
& \bar{\gamma}_{\Xi^{0}}^{(\pi)}=(D-F)^{2} \mu_{F}+\frac{4}{27} \mathcal{C}^{2} \mu_{C}+\frac{2}{9} \mathcal{C}(F-D) \mu_{T}, \\
& \bar{\gamma}_{\Xi-}^{(\pi)}=-\mu_{D}+\mu_{F}+\frac{1}{2}(D-F)^{2}\left(\mu_{D}+\mu_{F}\right)+\frac{2}{27} \mathcal{C}^{2} \mu_{C}+\frac{4}{9} \mathcal{C}(F-D) \mu_{T}, \\
& \bar{\gamma}_{\Lambda \Sigma^{0}}^{(\pi)}=-\frac{1}{\sqrt{3}} \mu_{D}+\frac{2}{3 \sqrt{3}} D\left(6 F \mu_{F}-D \mu_{D}\right)-\frac{4}{9 \sqrt{3}} \mathcal{C}^{2} \mu_{C}+\frac{1}{9 \sqrt{3}} \mathcal{C}(D+6 F) \mu_{T},
\end{aligned}
$$


for the pion loops,

$$
\begin{aligned}
\bar{\gamma}_{p}^{(K)=}= & \mu_{D}-\mu_{F}+\left(-\frac{1}{9} D^{2}+2 D F-F^{2}\right) \mu_{D}-(D-F)^{2} \mu_{F}-\frac{4}{27} \mathcal{C}^{2} \mu_{C} \\
& +\frac{2}{9} \mathcal{C}(3 D-F) \mu_{T}, \\
\bar{\gamma}_{n}^{(K)}= & \left(-\frac{7}{9} D^{2}+\frac{2}{3} D F+F^{2}\right) \mu_{D}+(D-F)^{2} \mu_{F}+\frac{4}{27} \mathcal{C}^{2} \mu_{C}-\frac{4}{9} \mathcal{C} F \mu_{T}, \\
\bar{\gamma}_{\Lambda}^{(K)}=- & \frac{1}{3} \mu_{D}+\left(\frac{1}{9} D^{2}+F^{2}\right) \mu_{D}-2 D F \mu_{F}+\frac{2}{9} \mathcal{C}^{2} \mu_{C}+\frac{2}{9} \mathcal{C}(D-3 F) \mu_{T}, \\
\bar{\gamma}_{\Sigma^{+}}^{(K)}=- & \mu_{D}-\mu_{F}+\left(\frac{1}{3} D^{2}+2 D F+\frac{1}{3} F^{2}\right) \mu_{D}-(D-F)^{2} \mu_{F}-\frac{28}{27} \mathcal{C}^{2} \mu_{C}+\frac{8}{9} \mathcal{C} D \mu_{T}, \\
\bar{\gamma}_{\Sigma^{0}}^{(K)}=- & \mu_{D}+\left(\frac{1}{3} D^{2}+\frac{1}{3} F^{2}\right) \mu_{D}+2 D F \mu_{F}-\frac{2}{9} \mathcal{C}^{2} \mu_{C}+\frac{2}{9} \mathcal{C}(D+F) \mu_{T}, \\
\bar{\gamma}_{\Sigma^{-}}^{(K)}=- & \mu_{D}+\mu_{F}+\left(\frac{1}{3} D^{2}-2 D F+\frac{1}{3} F^{2}\right) \mu_{D}+(D+F)^{2} \mu_{F}+\frac{16}{27} \mathcal{C}^{2} \mu_{C} \\
& +\frac{4}{9} \mathcal{C}(F-D) \mu_{T}, \\
\bar{\gamma}_{\Xi^{0}}^{(K)}= & \left(-\frac{7}{9} D^{2}-\frac{2}{3} D F+F^{2}\right) \mu_{D}-(D+F)^{2} \mu_{F}+\frac{8}{27} \mathcal{C}^{2} \mu_{C}-\frac{4}{9} \mathcal{C}(D+2 F) \mu_{T}, \\
\bar{\gamma}_{\Xi^{-}}^{(K)}=- & \mu_{D}+\mu_{F}+\left(-\frac{1}{9} D^{2}-2 D F-F^{2}\right) \mu_{D}+(D+F)^{2} \mu_{F}+\frac{16}{27} \mathcal{C}^{2} \mu_{C}+ \\
& \quad \frac{2}{9} \mathcal{C}(F-D) \mu_{T}, \\
\bar{\gamma}_{\Lambda \Sigma^{0}}^{(K)}=- & \frac{1}{\sqrt{3}} \mu_{D}+\frac{1}{\sqrt{3}}\left(D^{2}-3 F^{2}\right) \mu_{D}+\frac{2}{\sqrt{3}} D F \mu_{F}-\frac{2}{9 \sqrt{3}} \mathcal{C}^{2} \mu_{C}+\frac{4}{9 \sqrt{3}} \mathcal{C}(2 D+3 F) \mu_{T},
\end{aligned}
$$

for the kaon loops, and

$$
\begin{aligned}
& \bar{\gamma}_{p}^{(\eta)}=-\frac{1}{18}(D-3 F)^{2}\left(\mu_{D}+3 \mu_{F}\right), \\
& \bar{\gamma}_{n}^{(\eta)}=\frac{1}{9}(D-3 F)^{2} \mu_{D}, \\
& \bar{\gamma}_{\Lambda}^{(\eta)}=\frac{2}{9} D^{2} \mu_{D} \\
& \bar{\gamma}_{\Sigma^{+}}^{(\eta)}=-\frac{2}{9} D^{2}\left(\mu_{D}+3 \mu_{F}\right)-\frac{2}{9} \mathcal{C}^{2} \mu_{C}+\frac{4}{9} \mathcal{C} D \mu_{T}, \\
& \bar{\gamma}_{\Sigma^{0}}^{(\eta)}=-\frac{2}{9} D^{2} \mu_{D}+\frac{2}{9} \mathcal{C} D \mu_{T}, \\
& \bar{\gamma}_{\Sigma^{-}}^{(\eta)}=-\frac{2}{9} D^{2}\left(\mu_{D}-3 \mu_{F}\right)+\frac{2}{9} \mathcal{C}^{2} \mu_{C}, \\
& \bar{\gamma}_{\Xi^{0}}^{(\eta)}=\frac{1}{9}(D+3 F)^{2} \mu_{D}-\frac{2}{9} \mathcal{C}(D+3 F) \mu_{T}, \\
& \bar{\gamma}_{\Xi^{-}}^{(\eta)}=-\frac{1}{18}(D+3 F)^{2}\left(\mu_{D}-3 \mu_{F}\right)+\frac{2}{9} \mathcal{C}^{2} \mu_{C}, \\
& \bar{\gamma}_{\Lambda \Sigma^{0}}^{(\eta)}=\frac{2}{3 \sqrt{3}} D^{2} \mu_{D}+\frac{1}{3 \sqrt{3}} \mathcal{C} D \mu_{T},
\end{aligned}
$$

for the $\eta$ loops. All the loop coefficients include contributions from intermediate octet and decuplet states. The values of the coefficients neglecting all decuplet contributions are obtained trivially by setting $\mathcal{C}=0$. 


\section{References}

[1] S. Coleman and S.L. Glashow, Phys. Rev. Lett. 6 (1961) 423

[2] D.G. Caldi and H. Pagels, Phys. Rev. D10 (1974) 3739

[3] L-F. Li and H. Pagels, Phys. Rev. Lett. 26 (1971) 1204 ;

P. Langacker and H. Pagels, Phys. Rev. D10 (1974) 2904 ;

H. Pagels, Phys. Rep. 16 (1975) 219

[4] J. Gasser and H. Leutwyler, Phys. Rep. 87 (1982) 77

[5] J. Gasser, M. Sainio and A. Svarc, Nucl. Phys. B307 (1988) 779

[6] A. Krause, Helv. Phys. Acta. 63 (1990) 3

[7] E. Jenkins and A.V. Manohar, Phys. Lett. 255B (1991) 558, Phys. Lett. 259B (1991) 353

[8] E. Jenkins, Nucl. Phys. B368 (1992) 190, Nucl. Phys. B375 (1992) 561

[9] E. Jenkins and A.V. Manohar, Baryon Chiral Perturbation Theory, in Proceedings of the Workshop on "Effective Field Theories of the Standard Model," ed. U. Meissner, World Scientific (1992)

[10] M.N. Butler and M.J. Savage, UCSD Preprint UCSD/PTH 92-30 (1992) hep$\mathrm{ph} / 9209204$

[11] T.D. Cohen and W. Broniowski, Phys. Lett. 292B (1992) 5, and University of Maryland Preprints 92-193 and 92-225 (1992) [hep-ph/9208256-7]

[12] H.T. Diehl, et al., Phys. Rev. Lett. 67 (1991) 804

[13] M.N. Butler, M.J. Savage, and R.P. Springer, Strong and Electromagnetic Decays of the Baryon Decuplet, UCSD Preprint UCSD/PTH 92-37 (1992) hep-ph/9211247

[14] M.A.B. Bég, B.W. Lee and A. Pais, Phys. Rev. Lett. 13 (1964) 514;

C. Becchi and G. Morpurgo, Phys. Lett. 17 (1965) 352

[15] Review of Particle Properties, Phys. Rev. D45 (1992) VIII.16

[16] R.L. Jaffe and A.V. Manohar, Nucl. Phys. B337 (1990) 509 


\section{Figure Captions}

Fig. 1. Diagrams which produce the non-analytic $m_{q}^{1 / 2}$ contribution to the baryon magnetic moments. Dashed lines denote pions; single and double solid lines denote octet and decuplet baryons, respectively.

Fig. 2. Diagrams which produce the non-analytic $m_{q} \ln m_{q}$ contribution to the baryon magnetic moments (excluding the wavefunction renormalization graphs). Dashed lines denote pions; single and double solid lines denote octet and decuplet baryons, respectively. The photon vertices in (a) and (b) are from the octet baryon moment, in (c) from the decuplet magnetic moment, and in (d) and (e) from the decuplet-octet transition magnetic moment. 


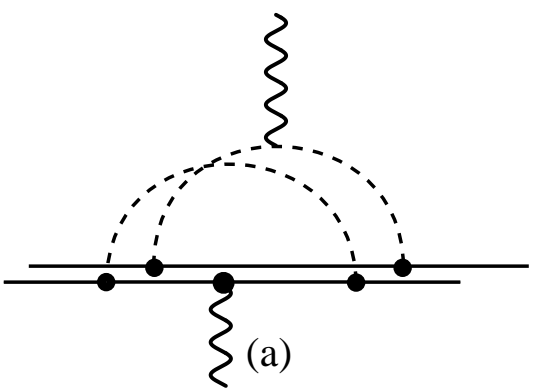

(a)

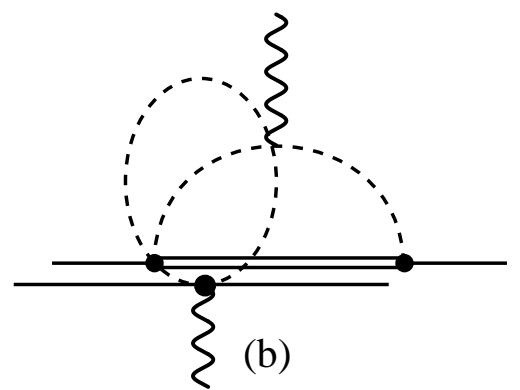

(b)

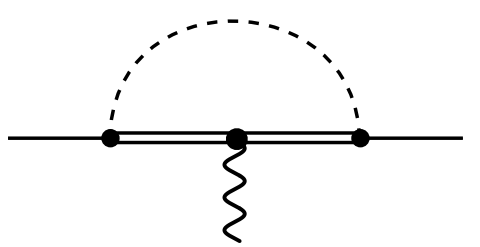

(c)

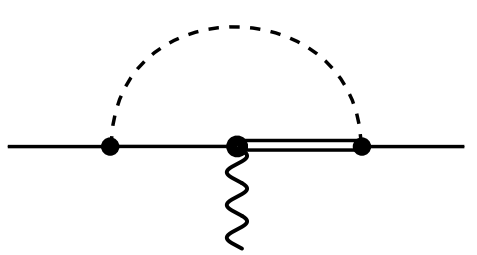

(d)

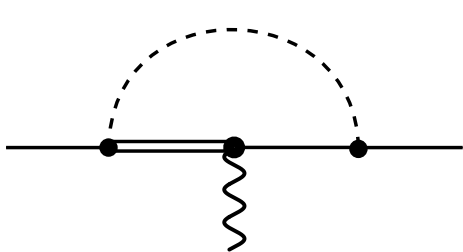

(e) 